\title{
Measurement of liquid sheet using laser tagging method by photochromic dye
}

\author{
Nurrina Binti Rosli $\cdot$ Kenji Amagai
}

Received: 1 August 2013 / Revised: 6 October 2014 / Accepted: 17 October 2014 / Published online: 18 November 2014

(C) The Author(s) 2014. This article is published with open access at Springerlink.com

\begin{abstract}
Liquid atomization system has been extensively applied as the most significant process in many industrial fields. In the internal combustion engine, the combustion phenomenon is strongly influenced by the spray characteristics of the fuel given by the atomization process. In order to completely understand the whole atomization process, a detail investigation of relations between the liquid jet characteristics and the breakup phenomenon is required. In this study, a non-intrusive method called as laser tagging method by photochromic dye has been developed with aim to study the breakup process of liquid sheet in detail, covering from the behavior in film until disintegrated into ligament and droplets. The laser tagging method by photochromic dye is based on a shift in the absorption spectrum of photochromic dye molecules tagged by ultraviolet laser. The shift results a color change at the tagged region of liquid containing the dye. In this study, the motions of the dye traces were analyzed as the liquid surface velocity. As a result, liquid sheet was found to keep its velocity constantly in film before suddenly increase around broken point. However, it then decreased after broken into droplets. By forming a set of four points of dye traces on the liquid sheet, the change of relative position of the set enabled the measurement of deformation and rotational motion of the liquid sheet. As a result, the normal strain of the liquid sheet parallel to the flow direction depended on the flow behavior of ligament formation.
\end{abstract}

N. B. Rosli $\cdot$ K. Amagai $(\bowtie)$

Department of Mechanical Science and Technology,

Gunma University, Kiryu 376-8515, Japan

e-mail: amagai@gunma-u.ac.jp

\section{Introduction}

Liquid atomization presents in many industrial fields. From pesticides spray in agricultural to internal combustion engine in transportation and power generation industry, liquid atomization system has been extensively applied as the most significant process to ensure their continuous operations.

In the internal combustion engine, for example, the fuel combustion of diesel engine plays an important role in the engine performance. However, the combustion phenomenon is strongly influenced by the spray characteristics of the fuel given by the atomization process. This has attracted attention from researchers to clarify the spray flow behavior. However, most of the studies carried out by the researchers merely described the spray characteristics caused by the atomization process. Therefore, in order to completely understand the whole atomization process, a detail investigation of relations between the liquid jet characteristics and the breakup phenomenon is required.

Various measurement methods on the breakup process of the spray flow have been developed in the past. However, the methods sometimes present performance limitations when applied to certain flow fields. For instance, laser Doppler velocimetry (LDV) and particle image velocimetry (PIV) are limited to precisely evaluate the spray flows which presents liquid sheet, since the irregular refraction of laser light will appear when it enter the liquid. Therefore, a proper measurement method is required to provide appropriate assessment for such spray flow.

In this paper, we applied a non-intrusive method called as laser tagging method by photochromic dye for measuring velocity distribution in liquid sheet. This technique is based on a shift in the absorption spectrum of photochromic dye molecules tagged by ultraviolet (UV) laser. The 
shift then results a color change at the tagged region of liquid containing the dye. Thus, the tagged region can be tracked to investigate the liquid behavior.

The usage of photochromic dye in experimental study was initially developed by Popovich and Hummel (1967). Since then, its uses have been widely applied to develop variety of research fields, especially in the flow field measurements. For instance, considering the difficulty in precisely predicting heat and mass transfer during drop formation and release, Johnson and Marschall (1986) utilized this method to investigate the flow mechanism for dispersed phase during drop formation and release in various flow fields. They stated the ability of this technique to provide precise velocity information.

Fogwell and Hope (1987) studied the properties and usefulness of photochromic dye in detail. They presented the mechanisms and theory of chemical changes in the properties of many types of photochromic dye. Their investigation especially in describing the dye concentration is greatly beneficial in using photochromic dye properly for the flow visualization. Agui Garcia and Hesselink (1989) also used the photochromic materials as the sensitizing agent to increase the spatial resolution in their holographic method of measuring velocity gradient. Their study finally improved the spatial resolution down to $100 \mu \mathrm{m}$ and resolutions in the velocity gradient measurement up to $3 \mathrm{~s}^{-1}$.

Kawaji (1998) described several sample experiments conducted by his research team in order to prove the benefit of this method in visualizing the two-phase flow. The sample experiments include the wavy-stratified, free-falling film, annular and slug flows. The investigation has reached better understanding of the flow structures and made supports and denial to some previous assumptions. Park et al. (1999) used this method to experimentally study a twodimensional steady flow in a curved vessel. They measured the velocity fields and also estimated the vorticity, wall shear stress and pressure fields. In order to ensure the measurement accuracy of the method, the numerical flow field calculations were also performed. The results of both methods were then compared to prove the validity of the laser tagging method by photochromic dye, and a good agreement was achieved.

The laser tagging method by photochromic dye was also applied by Koso et al. (2000) to visualize the photochromic dye patterns in order to investigate the turbulent mass mixing caused by a rising bubble in a still fluid in a circular pipe. They also investigated the reliability and problems occurred when using the concentration measurement by the dye. Kai et al. (2003) proved the successful of using this method to visualize particle movement in a two-dimensional fluidized catalyst bed. They measured the local velocity of particles around bubble and observed the bubble behavior without disturbing the flow pattern.
Homescu and Desevaux (2004) applied this method to determine the liquid-free surface velocity on curved surfaces. The ability was shown on the silicone oil film flowing around finned tubes. The advantages and limitations in using this method were also described. A bubble wake visualization was also done by Sanada et al. (2007) using the method. They used silicone oil solution of photochromic dye to visualize the flow structure in the rear part of a single rising bubble in a quiescent liquid. They also noted that appropriate photochromic dye must be assessed carefully in order to gain a successful application of this method.

We also referred the study of Hosokawa et al. (2009). They improved a photobleaching molecular tagging velocimetry to evaluate turbulence energy budget in bubbly flows. They improved the method for accurate evaluation of velocity gradients that reflected from the deformation of the shape of the tagged region in the fluid. The turbulence properties in single-phase and two-phase dilute bubbly flows in a square duct were measured.

In our previous research of creeping flow field around a circular cylinder (2011), the laser tagging method by photochromic dye has been successfully applied to estimate the velocity and vorticity fields. The result showed that eddy structure was found only in the cylinder wake, and its maximum velocity was close to the relative velocity between the flow and the circular cylinder. The vorticity fields were also described in detail.

In this study, we focus on the liquid sheet atomization as a fundamental study of the atomization phenomena. The laser tagging method by photochromic dye was developed with aim to study the breakup process of liquid sheet in detail, covering from the behavior in film until disintegrated into ligament and droplets. The motions of the dye traces tagged by UV laser were analyzed as the liquid surface velocity. By forming a set of four points of dye traces on the liquid sheet, the change of relative position of the set enabled the measurement of deformation and rotational motion. Moreover, the measurement of average thickness of the liquid sheet was also taken using light absorption method. Several interesting results were obtained and discussed in detail.

\section{Experimental apparatus and methodology}

\subsection{Experimental apparatus}

Figure 1 shows the schematic view of experimental apparatus which consists of the liquid sheet generation equipment, optical system and image analysis system.

Kerosene oil dissolved with 0.12 wt $\%$ of photochromic dye was used as the working fluid. The photochromic dye utilized in this study was 


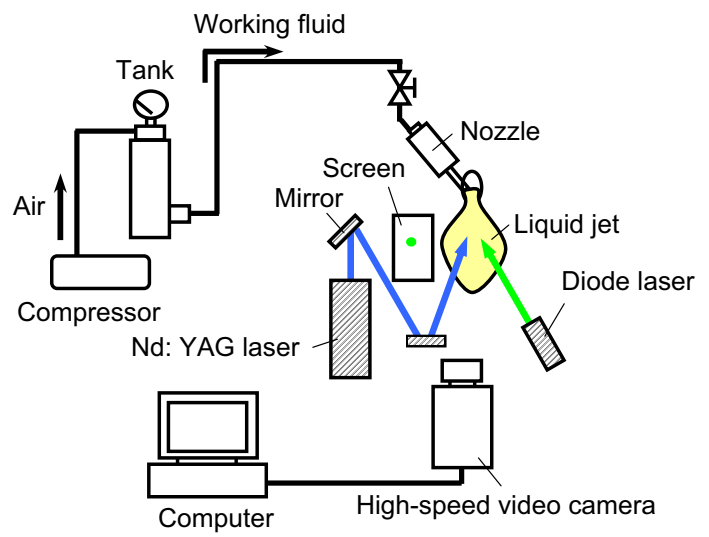

Fig. 1 Schematic view of experimental apparatus

(a)

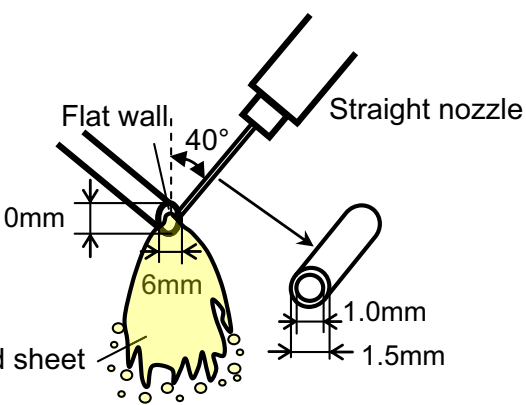

(b) Flat spray nozzle

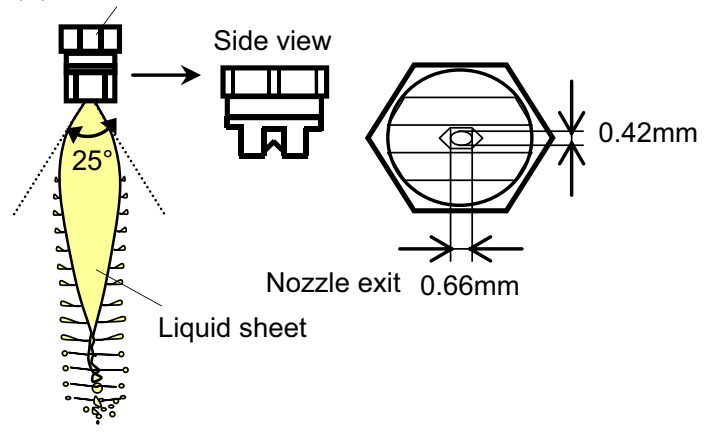

Fig. 2 Schematic view of a impingement spray and $\mathbf{b}$ flat spray

1,3,3-trimethylindolino-6'-nitrobenzophrylospiran. The transparent dye solution would change to a dark bluepurple color when irradiated by UV laser. In order to form the liquid sheet, the working fluid filled in the tank was compressed into the nozzle inlet with pressure of about $0.3 \mathrm{MPa}$.

As shown in Fig. 2, two types of liquid sheet spray were formed in this study, i.e., impingement spray and flat spray. The impingement spray was formed by applying impingement on the working fluid to a flat wall. The working fluid was injected from a straight nozzle (external diameter $=1.5 \mathrm{~mm}$; internal diameter $=1.0 \mathrm{~mm}$ ) that tilted $40^{\circ}$ to the flat wall. On the other hand, the flat spray was formed through injection of the working fluid from a commercial flat spray nozzle (orifice diameter $=0.66 \mathrm{~mm}$; spray angle $=25^{\circ}$ at $0.3 \mathrm{MPa}$ ). The sample images of both sprays are shown in Fig. 3.

The liquid sheet was then irradiated by pulses of UV laser from a Nd:YAG laser in order to form the photochromic dye traces. Beta barium borate (BBO) crystals were used to obtain the third harmonic light of the Nd:YAG laser (wavelength $\lambda=355 \mathrm{~nm}$ ). The laser pulse was $15 \mathrm{~ns}$ in duration and $100 \mathrm{~mJ} /$ pulse in energy.

The movement of the dye traces formed on the liquid sheet was then recorded by a high-speed video camera (NAC's Memrecam fx 6000). The camera provides framing rates in excess of 200,000 fps. For the impingement spray, the image was recorded with 5,000 fps at $512 \times 500$ pixel resolution. Thus, spatial resolution of actual space and temporal resolution were, respectively, $102 \mu \mathrm{m}$ and $0.2 \mathrm{~ms}$. For the flat spray, the image was recorded with $10,000 \mathrm{fps}$ at $512 \times 248$ pixel resolution. And thus, the spatial resolution of actual space and temporal resolution were, respectively, $123 \mu \mathrm{m}$ and $0.1 \mathrm{~ms}$.

Figure 4 shows the schematic view of the measuring section upon UV laser irradiation. As shown in the figure, a screen plate composed of four holes was placed on the UV laser passage in order to create a set of four points of dye traces on the liquid film with the same size of the holes (diameter $=0.8 \mathrm{~mm}$, pitch $=1.5 \mathrm{~mm}$ ). This is the smallest size of dye trace that we could effort for. The diameter of the dye traces could not be smaller than that as the high concentration of the dye traces color in the liquid sheet must be kept. Furthermore, although the pitch size must be smaller enough compared to the length scale of the flow phenomena, a relatively large pitch size between the dye traces is still needed in order to clearly track the movement of each dye trace for measuring the liquid deformation from the change of relative position of the dye traces. Therefore, this measurement length scale was the most appropriate size applicable this time.

As shown in Fig. 4, the origin was set as the impingement point and the nozzle exit, respectively, for the impingement spray and flat spray. $L_{c}$ represents the distance from the origin to the center position in between the four points of dye traces with the coordinate of the center position defined as $\left(x_{c}, y_{c}\right)$. Moreover, $L_{i}$ represents the distance from the origin to the center points of four dye traces with the coordinate of the center of each dye trace defined as $\left(x_{i}, y_{i}\right)$ with $i=1,2,3$ and 4 . The coordinate $\left(x_{i}, y_{i}\right)$ was digitized using the software provided with the camera.

The motion pictures captured by the camera were then transferred into a personal computer as a still image data. The sample image recorded by the camera is shown in 

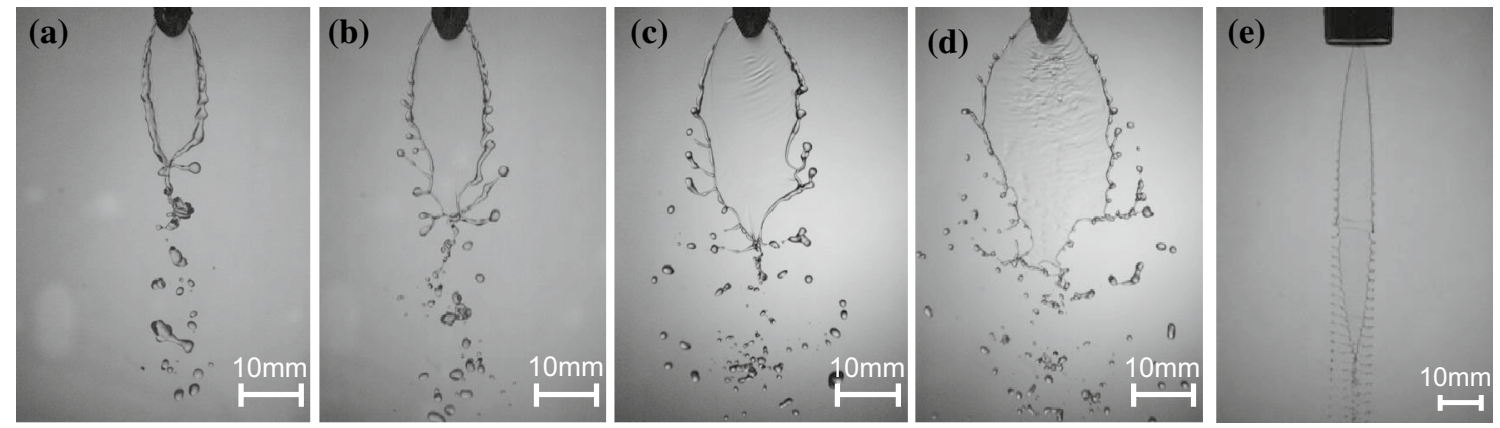

Fig. 3 Sample images of impingement spray at different flow rates: a $Q=1.98 \times 10^{-6} \mathrm{~m}^{3} / \mathrm{s}, \mathbf{b} Q=2.54 \times 10^{-6} \mathrm{~m}^{3} / \mathrm{s}, \mathbf{c} Q=3.14 \times 10^{-6} \mathrm{~m}^{3} / \mathrm{s}$, d $Q=3.66 \times 10^{-6} \mathrm{~m}^{3} / \mathrm{s}$ and e flat spray at flow rate $Q=1.98 \times 10^{-6} \mathrm{~m}^{3} / \mathrm{s}$

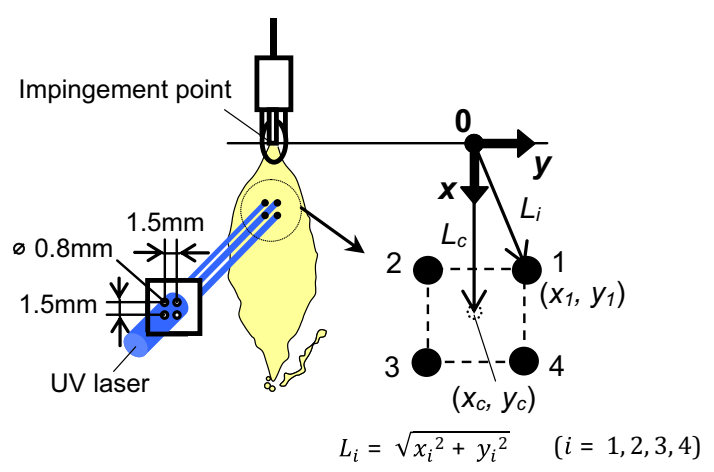

Fig. 4 Schematic view of measuring section upon UV laser irradiation

Fig. 5. The dark points on the liquid sheet represent the dye trace 1,2, 3 and 4 tagged by the UV laser irradiation as shown in the figure. In order to clearly spot the dye traces appearance, the image sharpness was enhanced using image analysis method.

For the impingement spray, the velocity, strain and rotational motion measurement was taken at position $L_{c}=10 \mathrm{~mm}$ under different flow rate $Q=1.98 \times 10^{-6} \mathrm{~m}^{3} / \mathrm{s}, 2.54 \times 10^{-6} \mathrm{~m}^{3} / \mathrm{s}, 3.14 \times 10^{-6} \mathrm{~m}^{3} / \mathrm{s}$ and $3.66 \times 10^{-6} \mathrm{~m}^{3} / \mathrm{s}$. For the flat spray, the measurement was taken only at position $L_{c}=13 \mathrm{~mm}$ under flow rate $Q=1.98 \times 10^{-6} \mathrm{~m}^{3} / \mathrm{s}$. For each experimental condition, the velocity, strain, rotational motion and thickness measurement was repeated in the same way 4-9 times in order to ensure the most accurate result. Further explanation on the measurement technique of velocity, deformation and thickness is provided in the next section.

\subsection{Velocity measurement of liquid sheet}

The movement of liquid sheet was tracked by digitizing the center points of dye traces, i.e., coordinate $\left(x_{i}\right.$,

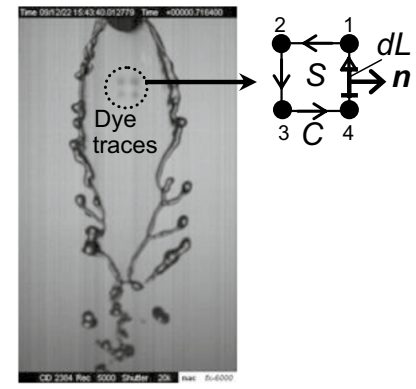

Fig. 5 Sample image recorded by high-speed video camera upon velocity measurement

Table 1 Initial position of center position $\left(x_{c}, y_{c}\right)$ in between four points of dye traces and center point $\left(x_{i}, y_{i}\right)$ of each dye trace

\begin{tabular}{lll}
\hline Position & Impingement spray & Flat spray \\
\hline$\left(x_{c}, y_{c}\right)$ & $(10,0)$ & $(13,0)$ \\
$\left(x_{1}, y_{1}\right)$ & $(9.25,0.75)$ & $(12.25,0.75)$ \\
$\left(x_{2}, y_{2}\right)$ & $(9.25,-0.75)$ & $(12.25,-0.75)$ \\
$\left(x_{3}, y_{3}\right)$ & $(10.75,-0.75)$ & $(13.75,-0.75)$ \\
$\left(x_{4}, y_{4}\right)$ & $(10.75,0.75)$ & $(13.75,0.75)$ \\
\hline
\end{tabular}

Unit: mm

$y_{i}$ ) from its initial position as shown in Table 1. Figure 6 shows the sample image of both sprays in which trajectory in the figure represents the movement of the dye traces.

As can be seen, the dye traces in impingement spray can be tracked until the liquid sheet broken into ligament and droplets. However, the dye traces in flat spray can be tracked until the liquid sheet disintegrated into ligament. The local velocity $V$ of each dye trace along the trajectory was then calculated using the following equation:

$V_{i}=\frac{\Delta L_{i}}{\Delta t} \quad(i=1,2,3,4)$ 
Fig. 6 Sample images of a impingement spray and $\mathbf{b}$ flat spray with trajectory of dye traces central point
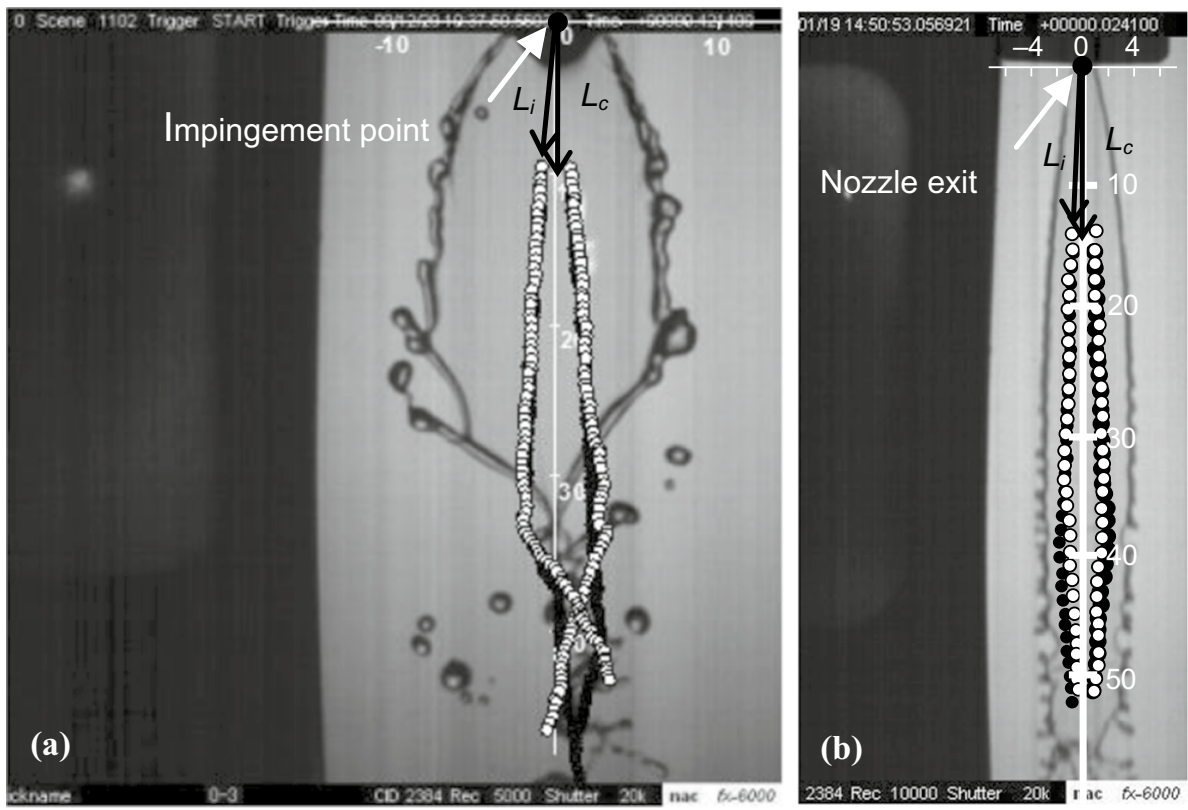

here, $\Delta L_{i}$ represents the displacement in two consecutive frames travelled by each dye trace $1,2,3$ and 4 . $\Delta t$ represents the inter-frame spacing for the motion pictures of the camera, i.e., 0.2 and $0.1 \mathrm{~ms}$, respectively, for the impingement spray and flat spray. The direction of the velocity vector was derived from the movement direction of dye trace. The velocity components, $u$ ( $x$ direction) and $v$ ( $y$ direction) were also derived.

\subsection{Evaluation method of normal strain, shear strain and rotational motion of fluid element of liquid sheet}

The deformation of the liquid sheet was estimated from the change of relative positions of four points of dye traces. In general, the normal strain in $x$ and $y$ directions, $\varepsilon_{x}$ and $\varepsilon_{y}$ are defined by the following equations:

$\varepsilon_{y}=\frac{\partial v}{\partial y}, \varepsilon_{x}=\frac{\partial u}{\partial x}$

where $u$ and $v$ are the velocity component in $x$ and $y$ direction. As defined here, the normal strains were described by the differential of velocity fields. However, in this study, the integral method was applied for the estimation of the normal strain. In the integral method, the average normal strain can be derived using the following equation (see "Appendix").

$\overline{\varepsilon_{x}} \cong \frac{1}{S} \iint_{S} \varepsilon_{x} \mathrm{~d} s=\frac{1}{S} \oint_{c} u n_{x} \mathrm{~d} l$

where $S$ is the area framed by the closed line $C, n_{x}$ is the $x$ direction component of normal vector $\boldsymbol{n}$ on the closed line $C$ and $\mathrm{d} l$ is the small line segment on the line as shown in
Fig. 5. Transformation from surface integral by Green's formula was applied here (see "Appendix"). If we assume that the closed line $C$ was formed by four points of dye traces, the average normal strain is described by:

$\overline{\varepsilon_{x}} \cong \frac{1}{S} \oint_{c} u n_{x} \mathrm{~d} l=\frac{1}{S} \sum_{(i j)=(12,23,34,41)} \frac{u_{i}+u_{j}}{2} n_{x i j} l_{i j}$

where $u_{i}$ and $u_{j}$ are the $x$ direction velocities of point $i$ and $j, n_{x i j}$ is an $x$ component of normal vector on line between $i$ and $j, l_{i j}$ is a length between point $i$ and $j$.

In the same way, the average normal strain of $y$ direction $\overline{\varepsilon_{y}}$ can also be derived using the following equation.

$$
\begin{aligned}
\overline{\varepsilon_{y}} \cong & \frac{1}{S} \iint_{s} \varepsilon_{y} \mathrm{~d} s=\frac{1}{S} \oint_{c} v n_{y} \mathrm{~d} l \\
& =\frac{1}{S} \sum_{(i j)=(12,23,34,41)} \frac{v_{i}+v_{j}}{2} n_{y i j} l_{i j}
\end{aligned}
$$

On the other hand, the average normal strain in $z$ direction $\overline{\varepsilon_{z}}$ can be derived from the equation of continuity as shown in the following equation.

$$
\begin{aligned}
\overline{\varepsilon_{z}} \cong & -\left(\overline{\varepsilon_{x}}+\overline{\varepsilon_{y}}\right) \\
& =-\oint_{c} v_{n} \mathrm{~d} l=-\frac{1}{S} \sum_{(i j)=(12,23,34,41)} \frac{v_{n i}+v_{n j}}{2} l_{i j}
\end{aligned}
$$

where $v_{n}$ is the magnitude of the normal vector $\boldsymbol{n}$ on the small line segment $d l$. Moreover, the shear strain $\gamma_{x y}$ is defined as the following equation.

$\gamma_{x y}=\frac{\partial v}{\partial x}+\frac{\partial u}{\partial y}$ 
Thus, the average shear strain $\overline{\gamma_{x y}}$ in this study can be derived using the following equation.

$$
\begin{aligned}
\overline{\gamma_{x y}} \cong & \frac{1}{S} \iint_{S} \gamma_{x y} \mathrm{~d} s=\frac{1}{S} \oint_{c}\left(v n_{x}+u n_{y}\right) \mathrm{d} l \\
& =\frac{1}{S} \sum_{(i j)=(12,23,34,41)}\left(\frac{v_{i}+v_{j}}{2} n_{x i j}+\frac{u_{i}+u_{j}}{2} n_{y i j}\right) l_{i j}
\end{aligned}
$$

On the other hand, the description of the rotational motion, i.e., vorticity $\omega$, is often used as the following equation.

$\omega=\frac{\partial v}{\partial x}-\frac{\partial u}{\partial y}$

Thus, the average rotational motion $\bar{\omega}$ in this study can be derived using the following equation:

$\bar{\omega} \cong \frac{1}{S} \iint_{S} \omega \mathrm{d} s=\frac{1}{S} \oint_{c} v_{t} \mathrm{~d} l=\frac{1}{S} \sum_{(i j)=(12,23,34,41)} \frac{v_{t i}+v_{t j}}{2} l_{i j}$

here, $v_{t}$ is the tangent direction component of the velocity vector, $v$.

\subsection{Measurement of average thickness of liquid sheet and the calibration procedure}

In this study, the time average liquid sheet thickness of impingement spray was also measured using a light absorption method. This method was conducted based on the light absorption of liquid containing a fluorescence dye.

The fluorescence dye used in this study was coumarin 153 , dissolved with $0.06 \mathrm{wt} \%$ of concentration to the test liquid that also containing the photochromic dye. A diode laser with wavelength $\lambda=408 \mathrm{~nm}$, i.e., around the maximum absorption wavelength of coumarin153, was used as the light source.

The light absorption method was referred from the wellknown theory of Beer-Lambert law which is described by the following equation:

$A=\log T=\log \left(I_{0} / I\right)=\varepsilon c \delta$

where $A$ is the measured absorbance, $T$ is the transmittance, $I_{0}$ is the intensity of the incident light, $I$ is the intensity of light passes through the liquid sheet, $\varepsilon$ is the molar extinction coefficient, $c$ is the molar concentration, and $\delta$ is the path length, i.e., the thickness of the liquid sheet. In order to determine the relation between the molar concentration of coumarin 153 and the extinction coefficient, a calibration procedure is needed.

Figure 7 illustrates the schematic view of the calibration procedure. The test equipment for the calibration procedure was made by sandwiching a known-thickness

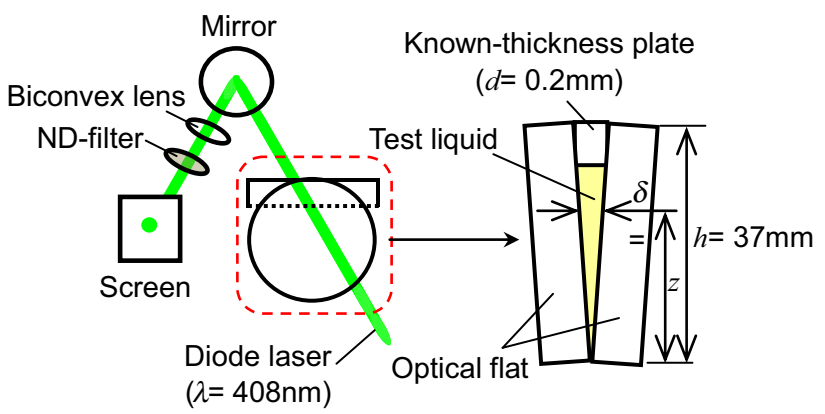

Fig. 7 Schematic view of calibration procedure of liquid sheet thickness measurement

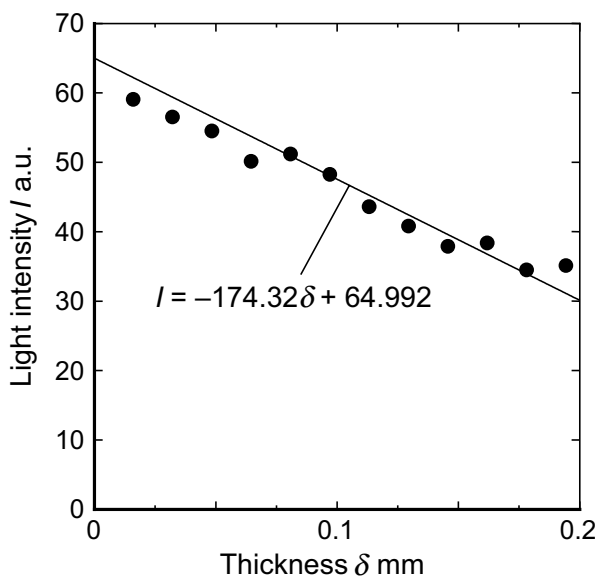

Fig. 8 Relation between light intensity and liquid sheet thickness

plate $(d=0.2 \mathrm{~mm})$ using two optical flat glasses. The plate was positioned on top of the two optical flat glasses while the remaining spaces were then filled up with the test liquid. The diode laser was irradiated to the test equipment in a range of distance from the bottom, $z=3 \mathrm{~mm}$ to $z=30 \mathrm{~mm}$. The transmitted light was projected on a screen, and the intensity was then quantified. The transmitted light intensity, $I$ was estimated to decrease with increasing liquid sheet thickness, $\delta$ indicated by the distance from the bottom, $z$.

The result of the calibration procedure is shown in Fig. 8. As can be seen, the liquid light intensity was found to linearly decrease with increasing liquid thickness. This calibration result was then used to determine the average thickness of liquid sheet.

Regarding the optical arrangement in the calibration procedure, a mirror was used to reflect the transmitted light to the screen while a biconvex lens and ND-filter were placed on the light path. The biconvex lens was used to focus the light. The ND-filter was used to filter the light intensity to ensure it does not exceed the measurable range of the camera. These devices were also employed in the actual experiment upon measuring the liquid sheet velocity as shown in Fig. 9. 


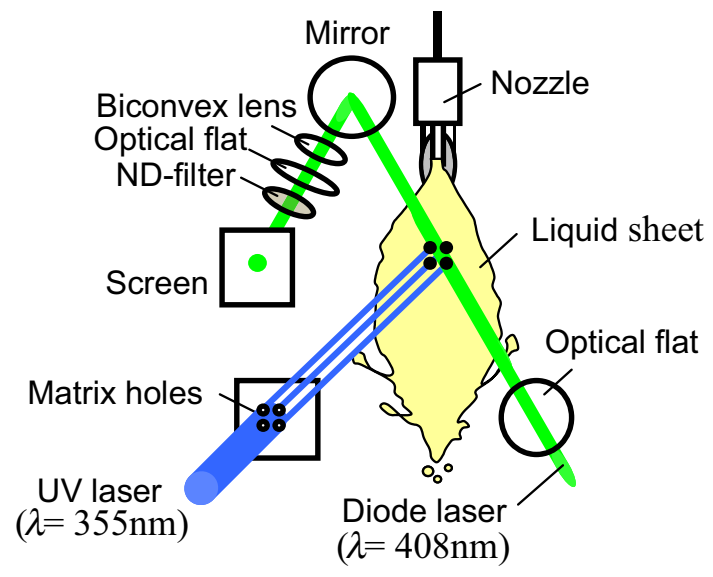

Fig. 9 Schematic view of measuring section upon diode laser irradiation

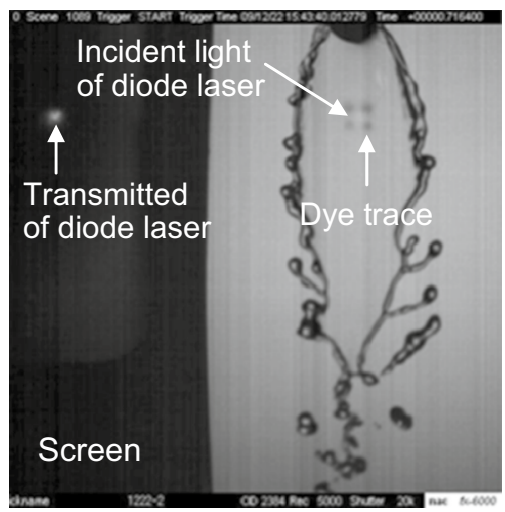

Fig. 10 Sample image recorded by high-speed video camera upon thickness measurement

The optical flat glasses used to make the test equipment in the calibration procedure were also used in the actual experiment in order to cancel the light reflection effect of the glass surface. The diode laser was led to the center position in between the four points of dye traces, i.e., coordinate $\left(x_{c}, y_{c}\right)$ at the initial position of the dye traces as shown in Table 1. From the product of thickness at this initial position and the area obtained from the photochromic dye marking method, the initial volume of liquid sheet can be obtained. Then, the change of liquid sheet thickness can be calculated by dividing the initial volume by the change of area. Thus, the validity of photochromic dye marking method can be estimated. The image of the transmitted light projected on the screen was recorded in the same frame of the image of dye traces. Figure 10 shows the sample recorded image.

Taking into account the intensity change of transmitted light due to the fluctuation of liquid sheet, the transmitted light reflected on the screen was captured with the liquid sheet images for its intensity quantifying. Therefore, only recorded image with such transmitted light was selected for the analysis procedure.

For the impingement spray, the thickness measurement was taken at different position $L_{c}=10,15,20,25$ and $30 \mathrm{~mm}$ under flow rate $Q=2.54 \times 10^{-6} \mathrm{~m}^{3} / \mathrm{s}$. For the flat spray, the measurement was taken only at position $L_{c}=13 \mathrm{~mm}$ under flow rate $Q=1.98 \times 10^{-6} \mathrm{~m}^{3} / \mathrm{s}$. For each experimental condition, the measurement was repeated in the same way 4-9 times in order to obtain the most accurate result. From this multiple times of measurement results, the averaged value was determined as the average thickness of liquid sheet.

\subsection{Estimation of measurement accuracy and resolution}

One source of weakness in this study which could have affected the measurement accuracy was the size of tagged area, i.e., four points of dye traces originally projected from the screen holes (diameter $=0.8 \mathrm{~mm}$, pitch $=1.5 \mathrm{~mm}$ ). This was the smallest size that can be operated in order to maintain concentration of the dye traces color which easily fades by the liquid flow being measured. However, it is needed to acknowledge that this size was relatively large compared to the measurement length scale in this study. Therefore, macroscopic deformation of liquid film is still yet to be achieved.

From the diameter of each dye trace tagged in the liquid film, the center position which was coordinated manually by eye vision can be estimated to slip around $\pm 0.02 \mathrm{~mm}$. Thus, the pitch size of the tagged area may also slip around $1.46-1.54 \mathrm{~mm}$. From here, the maximum measurement error of liquid surface area can be estimated as around $5 \%$. For liquid velocity, the measurement error was estimated by the movement distance of dye trace within a time difference per frame. Taking example from the impingement spray, the movement distance of dye trace was about $0.5 \mathrm{~mm}$ within $2.0 \times 10^{-4} \mathrm{~s}$. Thus, the maximum measurement error for impingement spray was found to be approximately $20 \%$.

In spite of those expected uncertainties, this study is beneficial to pave the way in experimentally measuring liquid sheet deformation. To our knowledge, such exploratory is still in critically needed but only been carried out in a small number of studies so far. Therefore, considerably more works must be done as recommendations to help us establishing a greater degree of accuracy and resolution for future study. What is mostly needed is to enhance the dye trace concentration and edge sharpness which were hardly achieved. The experimental design and tools in leading UV light to the liquid film for photochromic dye activation may be revised to meet such recommendation. Thus, the center of dye trace can be accurately coordinated for more reliable 
Fig. 11 Velocity change $\mathbf{a}, \mathbf{b}$ of impingement spray
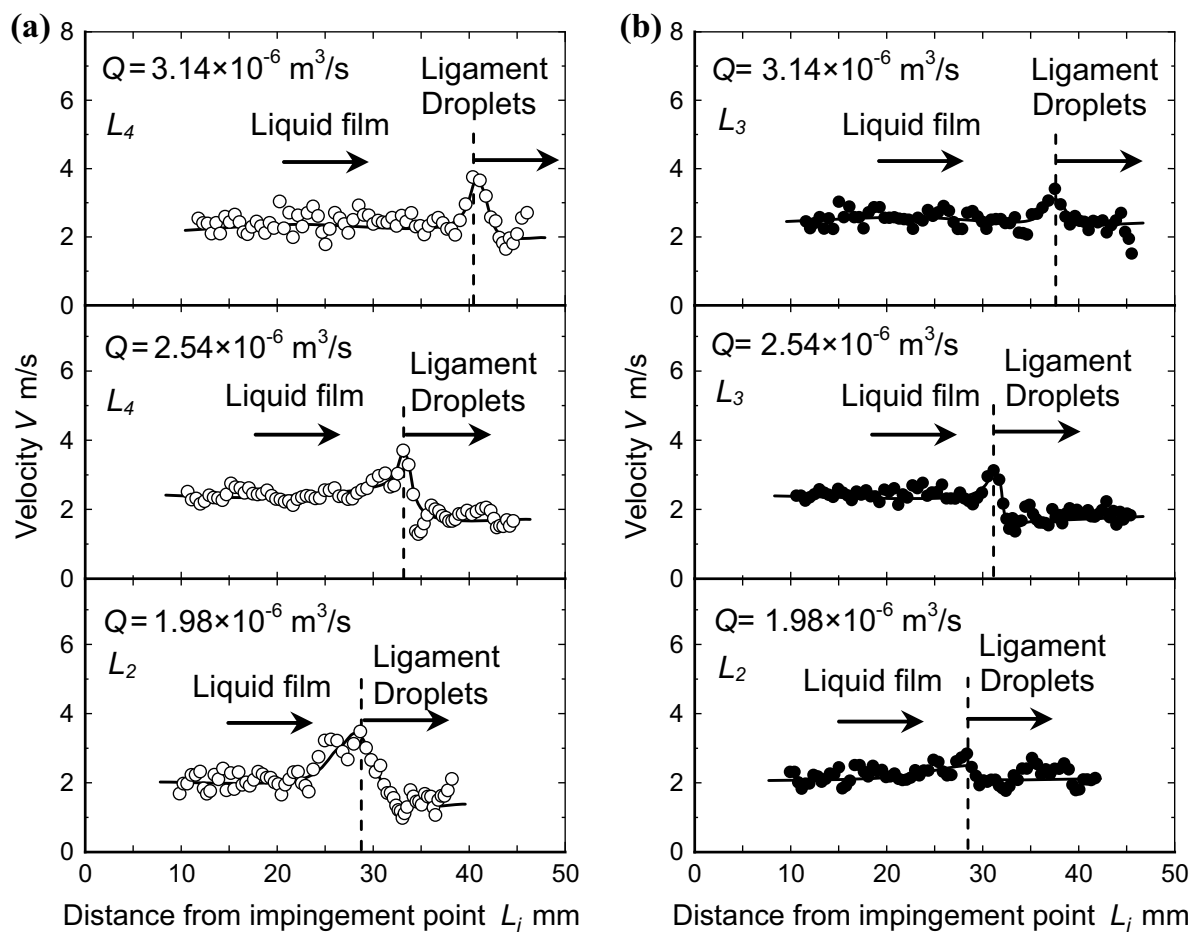

Fig. 12 Velocity change $\mathbf{a}, \mathbf{b}$ of flat spray

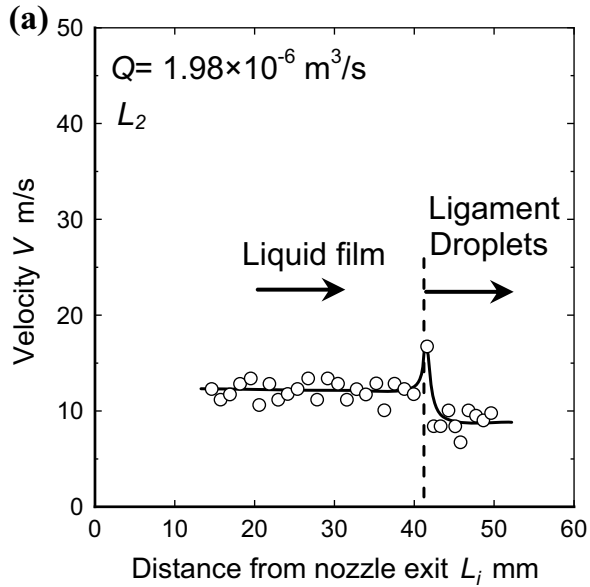

data and the scale of tagged area also can be reduced for a greater resolution. Furthermore, it would be more impressive if the number of dye traces bounding the tagged area can be increased to highlight the edge of bounded area for reliable liquid deformation measurement.

\section{Results and discussion}

\subsection{Velocity of liquid sheet}

Figures 11 and 12 show the result of local velocity change for one dye trace from its initial position, respectively, for impingement spray and flat spray as shown in Table 1. Both sprays were found two different trends of velocity change.

In the impingement spray, the velocity seemed to keep a constant value in the film before suddenly increase around the broken point as shown in Fig. 11a. The sudden increase indicates the moment of liquid sheet accumulated into the ligament. After broken into droplets, the velocity of the droplets was found to decrease with lower value than those in the liquid film. This can be attributed to the velocity component that arises in the opposite direction induced by the accumulation phenomenon caused by the effect of surface tension. However, the velocity difference did not 
Fig. 13 Total normal strain distribution of a impingement spray and $\mathbf{b}$ flat spray
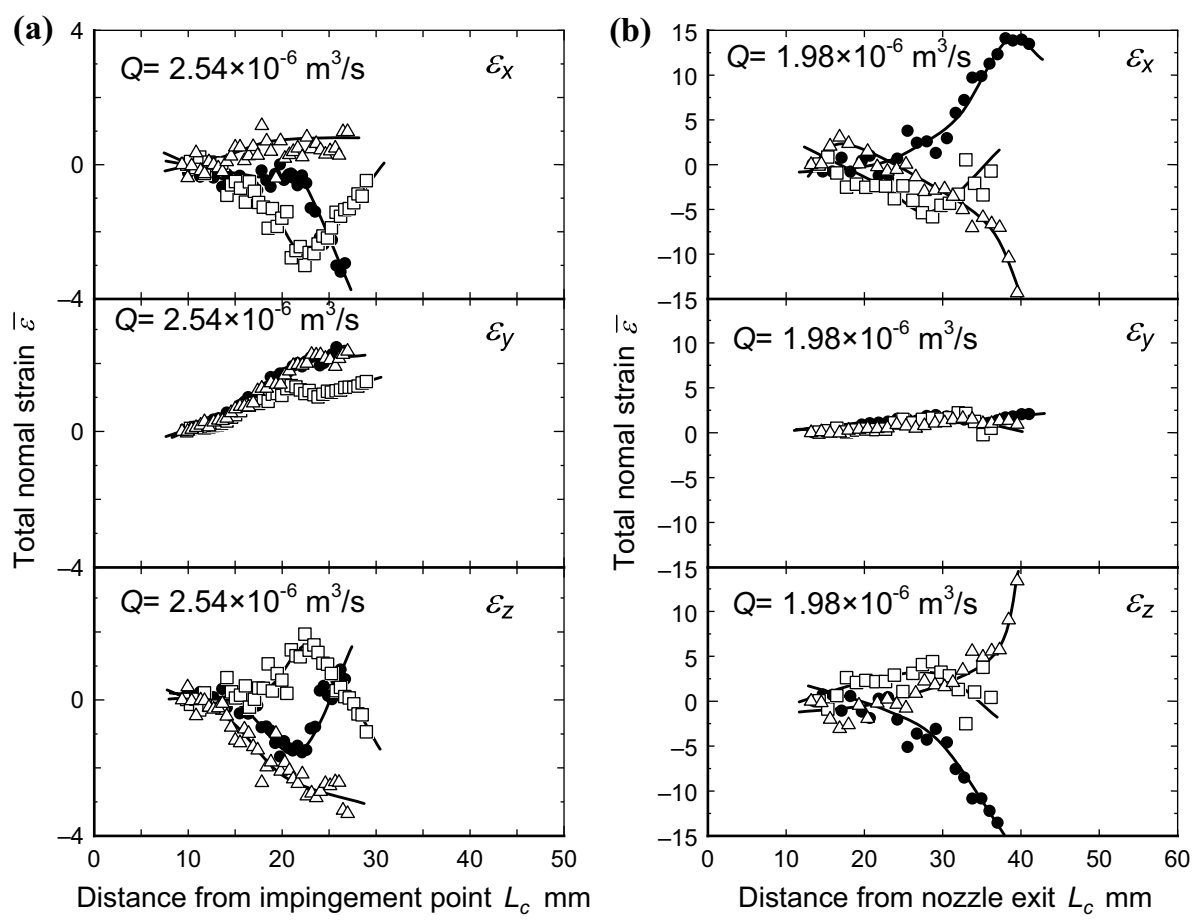

always significantly occur. As can be seen in Fig. 11b, the sudden increase as well as the velocity reduction in the droplets was seen very weak.

On the other hand, the increment and decrement of velocity change in flat spray were seen even lower than those in impingement spray. As shown in Fig. 12a, the sudden increase of liquid velocity of flat spray upon transition to ligament and droplets was found, but the peak of sudden increase was seen relatively lower than those in impingement spray. However, there was also case of the sudden increase that did not occur upon the transition into ligament and droplets as can be seen in Fig. 12b.

Comparing the results between the impingement spray and flat spray, the velocity of flat spray was found 5 times larger than those in impingement spray. In the impingement spray, the liquid sheet velocity was independent of the increase in flow rate. This can be attributed to the greater rate of attenuation due to the strong impingement of liquid sheet onto the wall, which then leads to widen area of the film. Thus, the distance between the broken point and impingement point became larger with increasing flow rate. These behaviors can be well confirmed by Fig. 3. In the flat spray, the position of liquid broken into ligaments at constant flow rate was seen around $L_{i}=40 \mathrm{~mm}$.

Moreover, a general look at Figs. 11 and 12 suggested a quite variable result of velocity change. For example, the uncertainty in the velocity of $L_{2}$ at flow rate $Q=1.98 \times 10^{-6} \mathrm{~m}^{3} / \mathrm{s}$ in Fig. 11a is found to range around $\pm 0.13 \mathrm{~m} / \mathrm{s}$ in the film and $\pm 0.23 \mathrm{~m} / \mathrm{s}$ in the ligament and droplets. The uncertainty probably caused by the difficulty

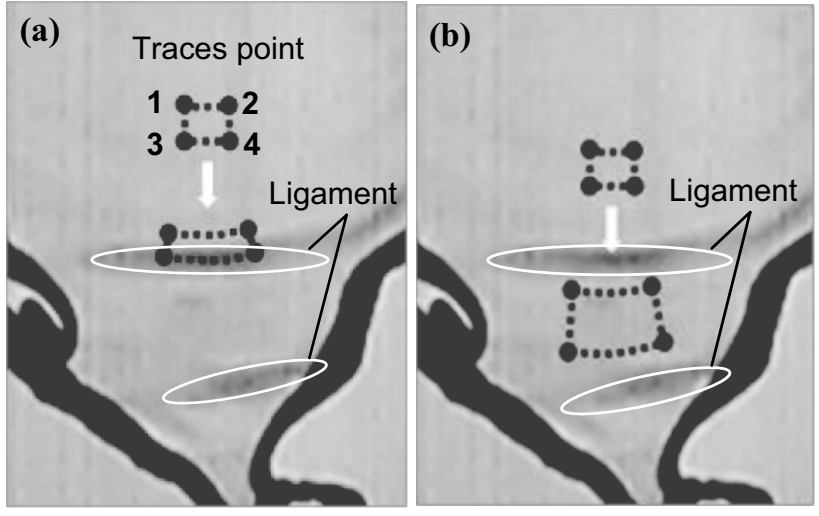

Fig. 14 Explanation of $\mathbf{a}$ decreasing trend and $\mathbf{b}$ increasing trend of normal strain by dye traces behavior

in coordinating accurately the center of the dye trace appeared along the trajectory due to its fading color further downstream.

\subsection{Total normal strain of liquid sheet}

Figure 13 shows the results of total normal strain distribution in $x, y$ and $z$ directions. The data were plotted as the total averaged value of the normal strain integrated by area starting from the initial position of the dye traces as shown in Table 1.

Further downstream, various trends of distribution were found under the same experimental condition. In the 
impingement spray, the normal strain in $x$ direction shows various changes. The reason can be described by the dye traces behavior as shown in the images of liquid sheet in Fig. 14. The ligaments in the figure was found around $L_{c}=20-25 \mathrm{~mm}$ in the liquid sheet. The decreasing trend indicates the moment when the dye traces longitudinally shrank and drawn into the ligament, which then leads to the rises of liquid sheet thickness. On the contrary, the increasing trend indicates the moment when the dye traces were drawn into a thinner film in between two ligaments. This description can be confirmed from the results of liquid sheet thickness around $L_{c}=25-30 \mathrm{~mm}$ in the next section.

For the normal strain in $y$ direction, all results were found to gradually increase. This can be attributed to the natural flow behavior of liquid sheet that extended transversely further downstream. Thus, the normal strain in $z$ direction was found to be mainly contrary with the results of $x$ direction.

In the flat spray, the normal strain in $x$ and $z$ directions exhibited the same trends like the impingement spray.

However, the normal strain in $y$ direction showed very weak increase. This can be attributed to the natural flow behavior that mainly acted downstream, as can be confirmed by Fig. 3. Comparing the results between the impingement spray and flat spray, the results of flat spray showed a maximum difference of 5 times larger than the impingement spray.

The uncertainty also occurred in the results of the total normal strain distribution. Taking example the data of $x$ direction total normal strain in Fig. 13a, it was found to range around \pm 0.15 .

\subsection{Total shearing strain and rotational motion of liquid sheet}

Figure 15 shows various trends of total shearing strain distribution appeared in the impingement spray. The data were also plotted as the total average value of the shearing strain integrated by area starting from the initial position of the dye traces as shown in Table 1. Total shearing strain means the total value of shearing strain experienced by the area bounded by the four points of dye traces from its initial appearance in the liquid film. It indicates how far the liquid body in the area bounded by the tags has been transformed from its original shape throughout its movement to downstream region.

As can be seen, the shearing strain of impingement spray was found to increasingly, decreasingly and slightly change from its initial appearance. From these various trends of distribution, typical representatives are shown in Fig. 16a, and its relationships with the position of the dye traces against $x$ and $y$ directions are shown in Fig. 16b-1 to b-3.

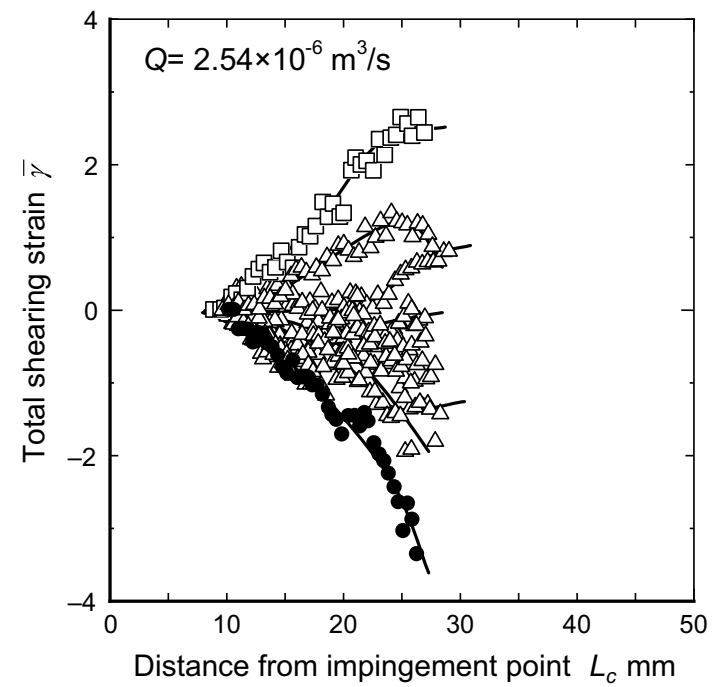

Fig. 15 Various trends of total shearing strain distribution appeared in impingement spray

As can be seen, the total shearing strain of impingement spray in Fig. 16a increased when the dye traces were frequently shifted from the centerline in $y$ direction as shown in Fig. 16b-1. Likewise, the total shearing strain decreased when the dye traces were frequently shifted from the centerline in $y$ direction as shown in Fig. 16b-3. Moreover, the total shearing strain did not much change when the dye traces stably move downstream with very few deviations from the centerline as shown in Fig. 16b-2. Thus, the instantaneous deviation of dye traces shifted from the centerline either in positive of negative $y$ direction was clarified. Taking example the data of Fig. 16a, the uncertainty was found to range around \pm 0.08 .

Figure 17 shows the rotational motion, i.e., vorticity distribution obtained from the averaged value integrated by area. It was found that little to no rotational motion took place in the liquid sheet of both sprays. Taking example the data of Fig. 17a, the uncertainty was found to range around $\pm 0.07 \mathrm{~s}^{-1}$.

\subsection{Average thickness of liquid sheet}

\subsubsection{Using light absorption method}

Figure 18 shows the result of liquid sheet thickness of impingement spray with increasing flow rate. As can be seen, the average thickness was found to be independent of the increase in flow rate, ranging around $154.3 \pm 3.2 \mu \mathrm{m}$. The results of liquid sheet thickness at different position $L_{c}$ are discussed in the next section. For flat spray, the data 


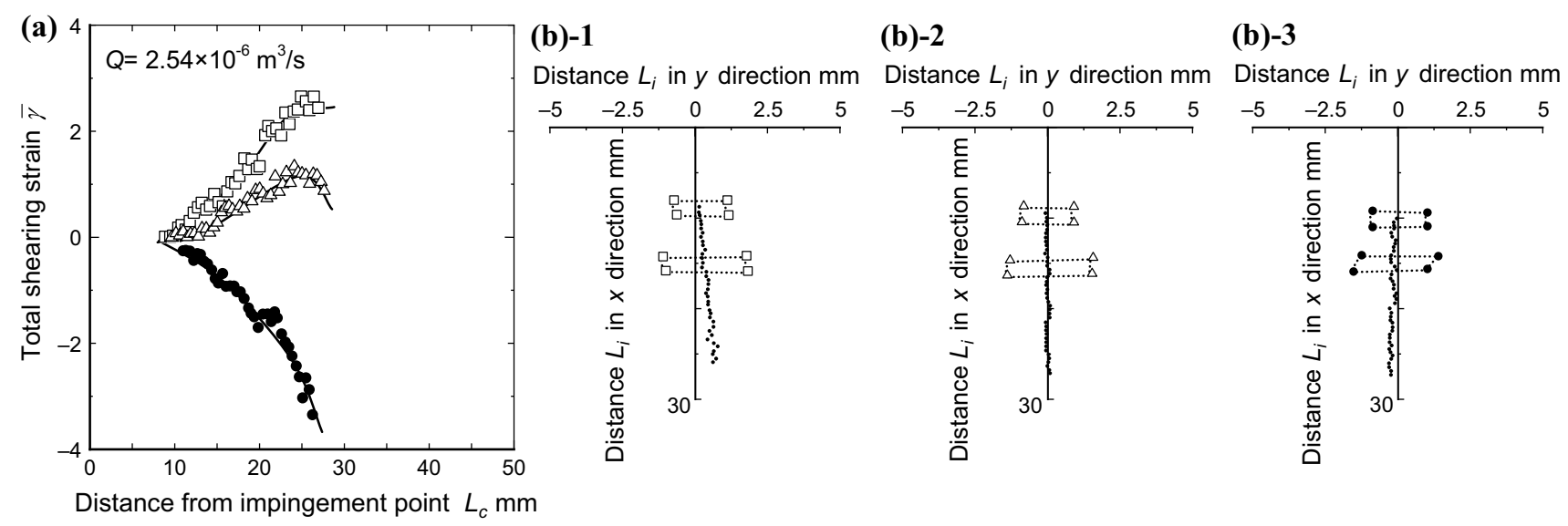

Fig. 16 a Total shearing strain distribution of impingement spray related with b position of dye traces against $x$ and $y$ direction

Fig. 17 Rotational motion of $\mathbf{a}$ impingement spray and $\mathbf{b}$ flat spray

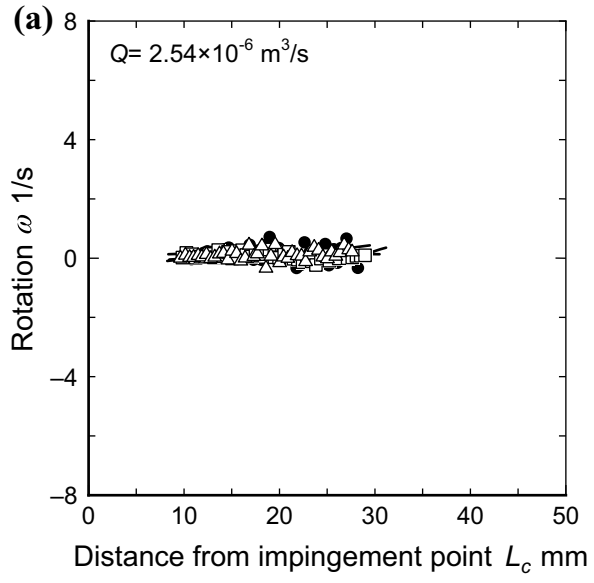

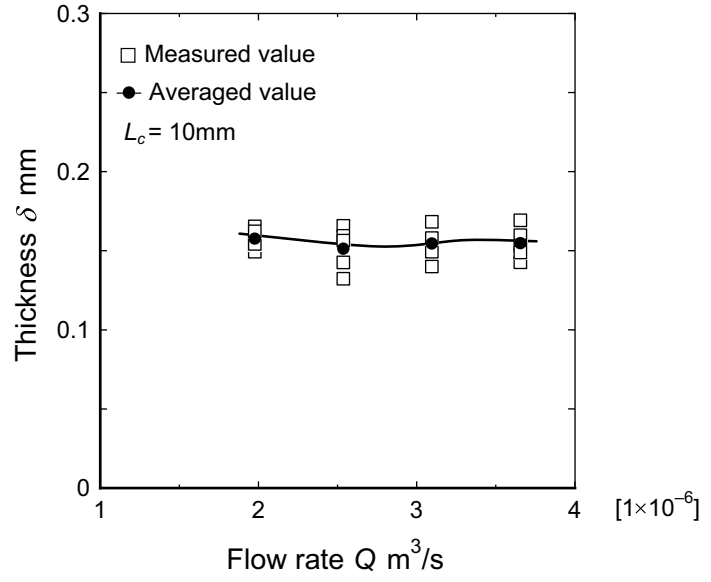

Fig. 18 Liquid sheet thickness of impingement spray with a increasing flow rate

were taken only at position $L_{c}=13 \mathrm{~mm}$ under flow rate $Q=1.98 \times 10^{-6} \mathrm{~m}^{3} / \mathrm{s}$ as mentioned before. The results are also discussed in the next section.

\subsubsection{From dye traces movement observation}

Figure 19 shows the change of area framed by the four points of dye traces. The results were found to gradually increase until approximately $L_{c}=25 \mathrm{~mm}$ and $L_{c}=30 \mathrm{~mm}$, respectively, for impingement spray and flat spray. These results were then used to obtain the change of liquid sheet thickness.

Figure 20 shows the comparison between the change of liquid sheet thickness obtained from the change of area as mentioned above and the liquid sheet thickness obtained from the light absorption method. For the impingement spray, the change of liquid sheet thickness was found to decrease until approximately $L_{c}=25 \mathrm{~mm}$, but then appeared to increase until the next $5 \mathrm{~mm}$. This tendency was seen to meet the results obtained from the light absorption method. As can be seen, the average liquid sheet thickness further downstream obtained from the light absorption method was found to decrease into $94.1 \pm 14.0 \mu \mathrm{m}$ at $L_{c}=25 \mathrm{~mm}$. 
Fig. 19 Area framed by dye traces of a impingement spray and $\mathbf{b}$ flat spray (a)
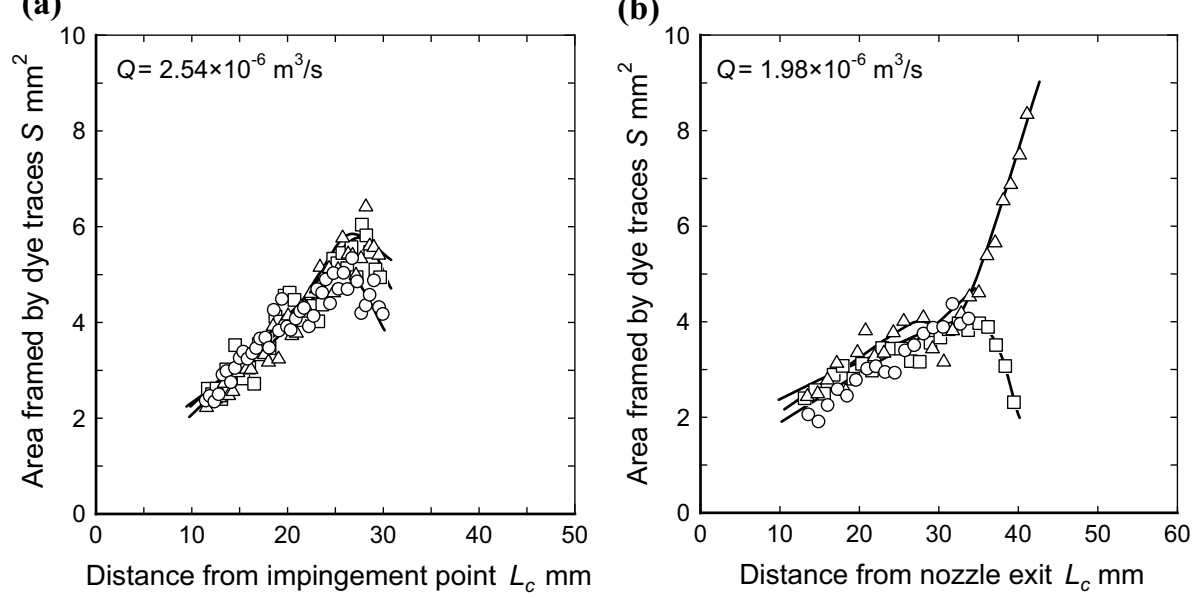

Fig. 20 Data comparison of liquid sheet thickness of a impingement spray and $\mathbf{b}$ flat spray

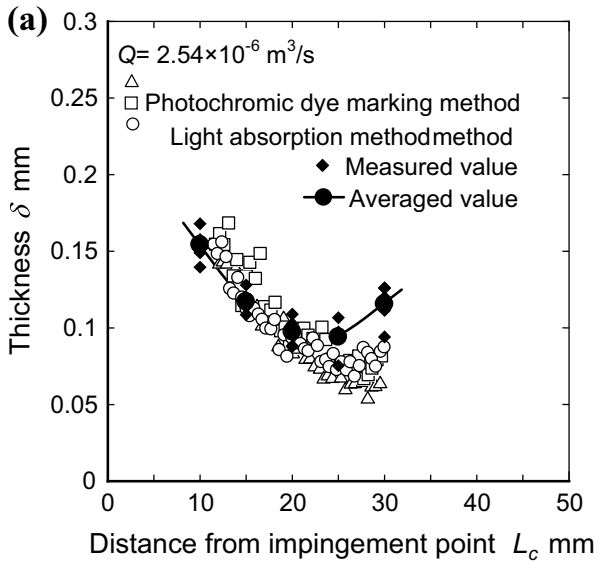

For the flat spray, the average liquid sheet thickness obtained from the light absorption method at $L_{c}=13 \mathrm{~mm}$ was around $36.6 \pm 12.2 \mu \mathrm{m}$. As can be seen, the change of liquid sheet thickness obtained from photochromic dye marking method at the same region was also ranging around the results obtained from light absorption method.

This similar tendency of liquid sheet thickness results between the two methods straighten the explanation of total normal strain distribution as mentioned before in Fig. 14. As can be seen, the liquid sheet thickness in Fig. 20 is found to vary around $L_{c}=25-30 \mathrm{~mm}$. This is the range of liquid sheet approaching the broken point in which the thickness in that region varied with the formation of ligament. Thus, a lower value of liquid sheet thickness at the region represents the moment when the liquid sheet drawn into a thinner film in between two ligaments as discussed in Fig. 14. Therefore, the validity of laser tagging method by photochromic dye was well confirmed.

Moreover, a closer look at the results of liquid sheet thickness of impingement spray obtained from the photochromic dye marking method suggests that further downstream the liquid sheet prior to the broken point became thinner up around $50-80 \mu \mathrm{m}$. For the flat spray, the liquid sheet prior to the broken point became thinner up around 13-25 $\mu \mathrm{m}$.

\section{Conclusions}

In this study, laser tagging method by photochromic dye has been developed with aim to study the breakup process of liquid sheet in detail, covering from the behavior in the film until disintegrated into ligament and droplets. The motions of the dye traces tagged by UV laser were analyzed as the liquid surface velocity. As a result, liquid sheet was found to keep its velocity constantly in film before suddenly increase around broken point. However, it then decreased after broken into droplets.

By forming a set of four points of dye traces on the liquid sheet, the change of relative position of the set enabled the measurement of deformation and rotational motion of the liquid sheet. As a result, the normal strain of the liquid sheet parallel to the flow direction was found to variously change depends on the liquid behavior against the ligament position. Whereby, the normal strain perpendicular 
to the flow direction was found to mainly increase further downstream due to natural behavior of the liquid sheet that extended transversely further downstream. Moreover, the shearing strain was found to occur only in the mainstream but also around the liquid edge. Also, little to no rotational motion occurred in the liquid sheet.

The measurement of average thickness of the liquid sheet has been taken using light absorption method. As a result, the average thickness of the liquid sheet was independent of the increase in flow rate. Further downstream, the thickness decreased until nearly reaching the broken point and then increased thereafter due to the liquid accumulation that occurs prior to the breakup process. Furthermore, the validity of laser tagging method by photochromic dye has also been well confirmed. It was proved by the similar tendency of liquid sheet thickness obtained from the change of area measured by the method and the thickness obtained from the laser absorption method.

Finally, although the uncertainties occurred in the measurement results, this study has successfully paved the way to experimentally better understand the liquid sheet deformation. Further works as recommended are required to establish a greater degree of accuracy and resolution.

Acknowledgments This research was funded by Japan Society for the Promotion of Science, JSPS KAKENHI (Grant No. 24560183). The author would also like to acknowledge Universiti Malaysia Pahang and Ministry of Higher Education of Malaysia for supporting and motivations.Open Access This article is distributed under the terms of the Creative Commons Attribution License which permits any use, distribution, and reproduction in any medium, provided the original author(s) and the source are credited.

\section{Appendix}

By setting $x$ and $y$ direction component of velocity vector $\boldsymbol{V}$ in two-dimensional flow as $u$ and $v$, the $x$ direction and $y$ direction normal strain rate $\varepsilon_{x}$ and $\varepsilon_{y}$ are given as:

$\varepsilon_{x}=\frac{\partial u}{\partial x}$

$\varepsilon_{y}=\frac{\partial v}{\partial y}$

The liquid sheet can be assumed as approximately twodimensional flow. In the case of liquid sheet thickness change with the deformation in two-dimensional direction, the normal strain rate in the thickness direction $\varepsilon_{z}$ can be defined as below equation using the equation of continuity:

$\varepsilon_{z}=\frac{\partial w}{\partial z}=-\left(\frac{\partial u}{\partial x}+\frac{\partial v}{\partial y}\right)=-\left(\varepsilon_{x}+\varepsilon_{y}\right)$

The shear strain rate, $\gamma$ can be defined as:

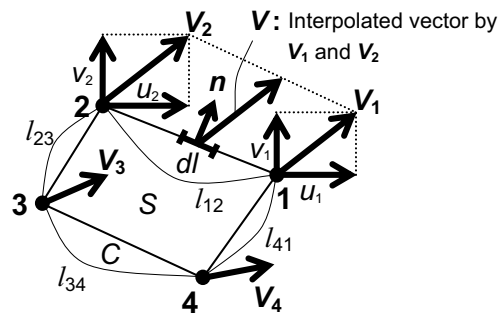

Fig. 21 Closed curve $C$ formed by dye traces

$\gamma=\frac{\partial v}{\partial x}+\frac{\partial u}{\partial y}$

And the vorticity $\omega$ can be defined as:

$\omega=\frac{\partial v}{\partial x}-\frac{\partial u}{\partial y}$

There are the integral theorems of vector fields known as Green's theorem, Gauss' theorem and Stokes' theorem. Although these theorems are commonly manifested as transformation formula of volume integral and surface integral in three-dimensional space, they are also applicable as transformation formula between the surface integral and volume integral over two-dimensional space.

Figure 21 shows a plane region $S$ bounded by the closed curve $C$ formed by the four points of dye traces. If we consider small linear element $d l$ on the line, the outwardpointing normal vector is expressed as $\boldsymbol{n}$, its $x$ and $y$ direction component is expressed as $n_{x}$ and $n_{y}$. Here, the Green's theorem over the arbitrary function $f(x, y)$ is given as:

$\iint_{S} \frac{\partial f}{\partial x} \mathrm{~d} S=\oint_{c} f n_{x} \mathrm{~d} l$

$\iint_{S} \frac{\partial f}{\partial y} \mathrm{~d} S=\oint_{c} f n_{y} \mathrm{~d} l$

here, by setting $f=u$ in Eq. (17) and $f=v$ in Eq. (18), i.e., the $u$ and $v$ direction component of the velocity $V$ for the dye traces as shown in Fig. 21,

$\iint_{S} \frac{\partial u}{\partial x} \mathrm{~d} S=\oint_{c} u n_{x} \mathrm{~d} l$

$\iint_{S} \frac{\partial v}{\partial y} \mathrm{~d} S=\oint_{c} v n_{y} \mathrm{~d} l$

are derived. Although these equations represent the integration by area $S$ of normal strain rate, $\varepsilon_{x}$ and $\varepsilon_{y}$ of Eqs. (12) and (13), they actually show the line integral by closed 
curve $C$ surrounding it. Here, the average normal strain rate, $\overline{\varepsilon_{x}}$ and $\overline{\varepsilon_{y}}$ can be obtained as:

$\overline{\varepsilon_{x}}=\frac{1}{S} \iint_{S} \frac{\partial u}{\partial x} \mathrm{~d} S=\frac{1}{S} \oint_{c} u n_{x} \mathrm{~d} l$

$\overline{\varepsilon_{y}}=\frac{1}{S} \iint_{S} \frac{\partial v}{\partial y} \mathrm{~d} S=\frac{1}{S} \oint_{c} v n_{y} \mathrm{~d} l$

Line integral of Eqs. (21) and (22) may be replaced as the following simple formula as below:

$\overline{\varepsilon_{x}} \cong \frac{1}{S} \oint_{c} u n_{x} \mathrm{~d} l=\frac{1}{S} \sum_{(i j)=(12,23,34,41)} \frac{u_{i}+u_{j}}{2} n_{x i j} l_{i j}$

$\overline{\varepsilon_{y}} \cong \frac{1}{S} \oint_{c} v n_{y} \mathrm{~d} l=\frac{1}{S} \sum_{(i j)=(12,23,34,41)} \frac{v_{i}+v_{j}}{2} n_{y i j} l_{i j}$

where we assumed that the closed line $C$ was formed by four points of dye traces. Moreover, $u_{i}, u_{j}$ and $v_{i}, v_{j}$ are, respectively, the $x$ and $y$ direction velocity measurement of point $i$ and $j . n_{x i j}$ and $n_{y i j}$ are, respectively, the $x$ and $y$ components of normal vector on line between $i$ and $j . l_{i j}$ is a length between point $i$ and $j$.

By summing Eqs. (19) and (20), the following equation is derived.

$\iint_{S}\left(\frac{\partial u}{\partial x}+\frac{\partial v}{\partial y}\right) \mathrm{d} S=\oint_{c}\left(u n_{x}+v n_{y}\right) \mathrm{d} l$

Here, the integrand of right-hand side is given as:

$u n_{x}+v n_{y}=v_{n}$

Thus, the Eq. (23) can be written as:

$\iint_{S}\left(\frac{\partial u}{\partial x}+\frac{\partial v}{\partial y}\right) \mathrm{d} S=\oint_{c} v_{n} \mathrm{~d} l$

This equation is also known as divergence theorem of Gauss. Similarly, the average normal strain rate in the thickness direction $\overline{\varepsilon_{z}}$ of Eq. (14) can be obtained as:

$$
\begin{aligned}
\overline{\varepsilon_{z}} & =-\frac{1}{S} \iint_{S}\left(\frac{\partial u}{\partial x}+\frac{\partial v}{\partial y}\right) \mathrm{d} S=-\frac{1}{S} \oint_{c} v_{n} \mathrm{~d} l \\
& =-\frac{1}{S} \sum_{(i j)=(12,23,34,41)} \frac{v_{n i}+v_{n j}}{2} l_{i j}
\end{aligned}
$$

where $v_{n}$ is the magnitude of the normal vector $\boldsymbol{n}$ on the small line segment $\mathrm{d} l$.

Next, by setting $f=v$ in Eq. (17) and $f=u$ in Eq. (18), the following equations are derived:
$\iint_{S} \frac{\partial v}{\partial x} \mathrm{~d} S=\oint_{c} v n_{x} \mathrm{~d} l$

$\iint_{S} \frac{\partial u}{\partial y} \mathrm{~d} S=\oint_{c} u n_{y} \mathrm{~d} l$

By summing the Eq. (29) and Eq. (30), the following equation is derived.

$\iint_{S}\left(\frac{\partial v}{\partial x}+\frac{\partial u}{\partial y}\right) \mathrm{d} S=\oint_{c}\left(v n_{x}+u n_{y}\right) \mathrm{d} l$

The left-hand side represents the surface integral of shear strain rate, $\gamma$. Thus, the average shear strain rate $\bar{\gamma}$ can be obtained as:

$$
\begin{aligned}
\bar{\gamma} & =\frac{1}{S} \iint_{S}\left(\frac{\partial v}{\partial x}+\frac{\partial u}{\partial y}\right) \mathrm{d} S=\frac{1}{S} \oint_{c}\left(v n_{x}+u n_{y}\right) \mathrm{d} l \\
& =\frac{1}{S} \sum_{(i j)=(12,23,34,41)}\left(\frac{v_{i}+v_{j}}{2} n_{x i j}+\frac{u_{i}+u_{j}}{2} n_{y i j}\right) l_{i j}
\end{aligned}
$$

By subtracting Eq. (30) from Eq. (29), the below equation is derived.

$\iint_{S}\left(\frac{\partial v}{\partial x}-\frac{\partial u}{\partial y}\right) \mathrm{d} S=\oint_{c}\left(v n_{x}-u n_{y}\right) \mathrm{d} l$

Here, the normal vector $\boldsymbol{n}$ is given as:

$n=\left(n_{x}, n_{y}\right)=\left(\frac{\mathrm{d} y}{\mathrm{~d} S},-\frac{\mathrm{d} x}{\mathrm{~d} S}\right)$

Thus, the integrand of right-hand side is given as:

$v n_{x}-u n_{y}=v \frac{\mathrm{d} y}{\mathrm{~d} S}+u \frac{\mathrm{d} x}{\mathrm{~d} S}$

Unit tangent vector $\boldsymbol{t}$ is given as:

$t=\left(t_{x}, t_{y}\right)=\left(\frac{\mathrm{d} x}{\mathrm{~d} S},-\frac{\mathrm{d} y}{\mathrm{~d} S}\right)$

Using unit tangent vector $\boldsymbol{t}$ into Eq. (35), below equations are derived.

$v n_{x}-u n_{y}=v t_{y}+u t_{x}=v_{t}=v \cdot t$

Here, $v_{t}$ represents the tangent direction component of velocity vector, $\boldsymbol{v}$. Thus, the Eq. (33) can be written as:

$\iint_{S}\left(\frac{\partial v}{\partial x}-\frac{\partial u}{\partial y}\right) \mathrm{d} S=\oint_{c} v_{t} \mathrm{~d} l$

This equation is called as two-dimensional Stokes' theorem. The left-hand side represents the surface integral of vorticity, $\omega$. Thus, the average vorticity $\bar{\omega}$ can be obtained as: 


$$
\begin{aligned}
\bar{\omega} & =\frac{1}{S} \iint_{S}\left(\frac{\partial v}{\partial x}-\frac{\partial u}{\partial y}\right) \mathrm{d} S=\frac{1}{S} \oint_{c} v_{t} \mathrm{~d} l \\
& =\frac{1}{S} \sum_{(i j)=(12,23,34,41)} \frac{v_{t i}+v_{t j}}{2} l_{i j}
\end{aligned}
$$

Thus, the average area of normal strain rate, shear strain rate and vorticity can be obtained from the linear integral of closed curve $C$.

\section{References}

Agui Garcia JC, Hesselink L (1989) Application of holography to the measurement of velocity gradient in fluid flows sensitized with photochromic dyes. Phys Fluids A-2:688-699

Fogwell TW, Hope CB (1987) Photochrome dye tracing in water flows. Exp Heat Transf 1-2:141-154

Homescu D, Desevaux P (2004) Laser photochromic dye activation technique for the measurement of liquid free surface velocity on the curved surfaces. Opt Lasers Eng 41:879-888

Hosokawa S, Fukunaga T, Tomiyama A (2009) Application of photobleaching molecular tagging velocimetry to turbulent bubbly flow in a square duct. Exp Fluids 47:745-754
Johnson G, Marschall E (1986) On the temperature jump in liquid-liquid direct-contact heat exchangers. Int $\mathrm{J}$ Multiph Flow 12-1:127-133

Kai T, Kanda T, Takahashi T, Kawaji M (2003) Application of photochromic dye to the measurement of particle movement in a fluidized bed. Powder Technol 129:22-29

Kawaji M (1998) Two-phase flow measurements using a photochromic dye activation technique. Nucl Eng Des 184:379-392

Koso T, Nakayama H, Kuniba Y, Hayami H (2000) Turbulent mass mixing in a bubble wake by using photochromic tracer. In: Proceedings of the annual conference of JSME vol 4, pp 223-224 (Japanese)

Park H, Moore JA, Trass O, Ojha M (1999) Laser photochromic velocimetry estimation of the vorticity and pressure field twodimensional flow in a curved vessel. Exp Fluids 26:55-62

Popovich AT, Hummel RL (1967) A new method for non-disturbing turbulent flow measurements very close to a wall. Chem Eng Sci $22-1: 21-25$

Rosli N, Toyooka Y, Amagai K (2011) Velocity and vorticity measurement in flow field using laser tagging method by photochromic dye. J JSME 11:147-152

Sanada T, Shirota M, Watanabe M (2007) Bubble wake visualization by using photochromic dye. Chem Eng Sci 62:7264-7273 